\title{
The enigmatic brown dwarf candidate [KG2001] 102 in the Chamaeleon I cloud: Is it a multiple system?^
}

\author{
P. Persi ${ }^{1}$, M. Tapia ${ }^{2}$, M. Gómez ${ }^{3}$, M. Roth ${ }^{4}$, and A. R. Marenzi ${ }^{1}$ \\ ${ }^{1}$ Istituto Astrofisica Spaziale e Fisica Cosmica, CNR, via fosso del cavaliere 100, 00133 Roma, Italy \\ e-mail: persi@rm.iasf.cnr.it \\ 2 Instituto de Astronomía, UNAM, Apartado Postal 877, 22830 Ensenada, BC Mexico \\ 3 Observatorio Astronómico de Córdoba, Laprida 854, 5000 Córdoba, Argentina \\ 4 Las Campanas Observatory, Carnegie Institution of Washington, Casilla 601, La Serena, Chile
}

Received 14 June 2004 / Accepted 11 October 2004

\begin{abstract}
We present deep $I J H K_{\mathrm{s}}$ and $\mathrm{H}_{2}$ images of a region in the Chamaeleon I dark cloud containing the very low-mass young object [KG2001]102, in an attempt to search for multiplicity and molecular outflow in the vicinity of this object. No lowvelocity shocked structures were detected in our $\mathrm{H}_{2}$ image. The broad-band and narrow-band images show that [KG2001]102 is composed of four objects within a radius of $\sim 2$. The brightest component [KG2001]102 A shows near-IR excess emission and its mass is estimated to be in the range 33 to $55 M_{\text {Jup }}$, depending on which model is adopted. Red spectra were obtained of the two fainter components B and C. The spectrum of the former suggests a K7V spectral type while the spectrum of component $\mathrm{C}$ is too noisy to allow a reliable classification but rules out a late $\mathrm{M}$-type. The three faint components $(\mathrm{B}, \mathrm{C}$ and $\mathrm{D})$ have $I J H K$ colors that suggest a much later spectral type, with extinctions similar to other members of the cloud. The computed probability of randomly finding a pair of field stars like [KG2001]102 AB is $1.5 \times 10^{-3}$ while for a triple optical system like [KG2001]102 ABC, it would be $3.8 \times 10^{-5}$ and more than an order of magnitude lower for finding a quadruple system like this by chance projection.
\end{abstract}

Key words. stars: low-mass, brown dwarfs - stars: formation - infrared: stars

\section{Introduction}

Although in recent years a number of sub-stellar systems have been discovered in nearby star forming regions (e.g. Wilking et al. 1999; Persi et al. 2000; Comerón et al. 2000; Saffe et al. 2003), very little is known about their formation processes. Mid-infrared observations have shown the presence of circumstellar dust around these objects (eg. Persi et al. 2000; Comerón et al. 2000) and very recently, Klein et al. (2003) detected cold dust around two young brown dwarfs (YBDs) from submm/mm observations. Natta \& Testi (2001) and Pascucci et al. (2003) analyzed the spectral energy distribution of two bona-fide YBDs (Cha $\mathrm{H} \alpha 1$ and CFHT-BD-Tau 4) and suggested that the dust around them is distributed in the form of disks. This would imply that the formation mechanism of sub stellar systems is analogous to that of $\mathrm{T}$ Tauri stars. If true, YBDs would produce molecular outflows detectable through $\mathrm{H}_{2}$ line emission during their accretion phases.

Another mechanism proposed by Reipurth \& Clarke (2001) and supported by recent hydrodynamical star formation calculations by Bate et al. (2002) is that brown dwarfs (BDs) form

^ Based on observations collected at Las Campanas Observatory (Chile) via the fragmentation of dense molecular gas in unstable multiple systems and that they are ejected from non-hierarchical systems before they are able to accrete to stellar masses. Several companions to bona-fide and candidate YBDs have been proposed by Neuhäuser et al. (2002) in the Chamaeleon I (Cha I) dark cloud. This underlines the importance of searching for multiplicity and molecular jets in YBDs in order to understand the mechanisms of their formation.

Here, we report the results of deep and high spatial resolution near-infrared images of an area towards the nearby dark cloud and star formation region Chamaeleon I, at a distance of $160 \mathrm{pc}$. The observed region contains the candidate substellar object [KG2001]102. This source shows conspicuous $\mathrm{H} \alpha$ line emission in its spectrum (Saffe et al. 2003), confirming its membership of the dark cloud. In particular, [KG2001]102 which was discovered recently by Kenyon \& Gómez (2001) during a near-IR survey of the Chamaeleon I (Cha I) cloud, appears to be a multiple system.

In Sect. 2, we describe the observations and in Sect. 3 we present the results and discussions with emphasis on whether [KG2001]102 is a physical multiple system. We estimate the luminosity and mass of the brightest component and present its spectral energy distribution. 

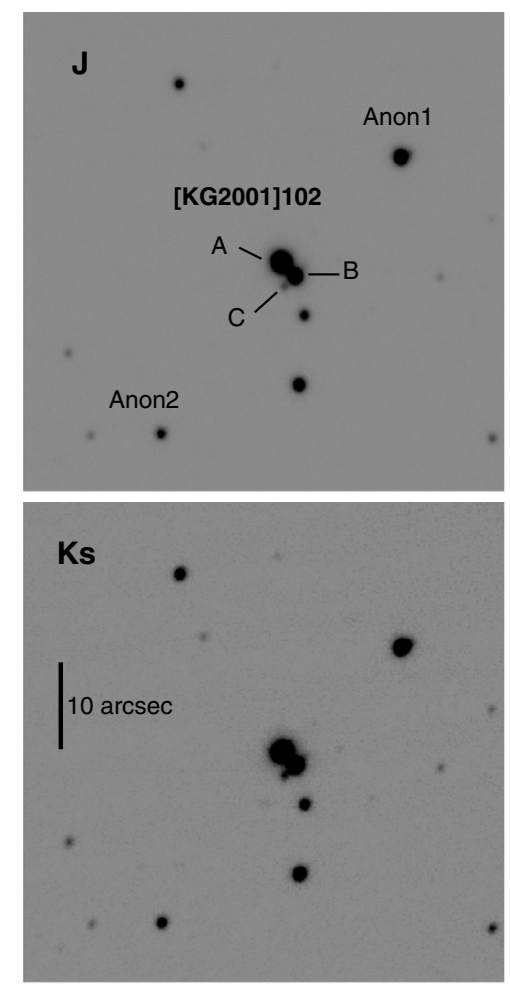
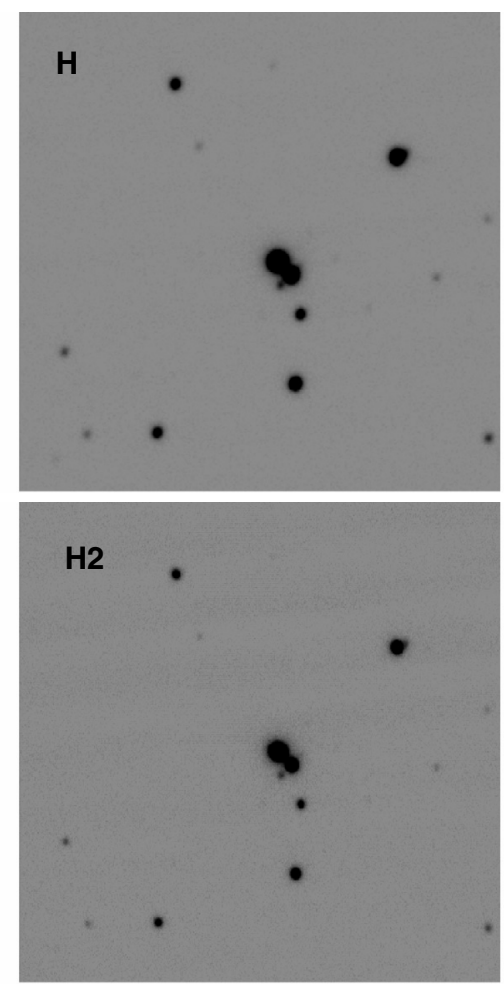

Fig. 1. $J H K_{\mathrm{s}}$ and $\mathrm{H}_{2}$ images centered at $\alpha=11^{\mathrm{h}} 09^{\mathrm{m}} 49^{\mathrm{s}} 5 ; \delta=-77^{\circ} 31^{\prime} 20^{\prime \prime}$ (J2000) containing the source [KG2001]102 in Cha I. North is up and east is to the left.

\section{Observations and data reduction}

\subsection{Imaging}

We obtained deep and high spatial resolution broad-band $J H K_{\mathrm{s}}$ images complemented with an $\mathrm{H}_{2}$ narrow-band image $\left(\lambda_{\circ}=\right.$ $2.149 \mu \mathrm{m} ; \Delta \lambda=0.0224 \mu \mathrm{m})$ of a region of the Chamaeleon I dark cloud that contains the candidate YBD [KG2001]102 (Saffe et al. 2003). The observations were made on the night of 17 May 2003 using the near-infrared camera PANIC (Persson's Auxiliary Nasmyth Infrared Camera) attached to the MagellanClay $6.5 \mathrm{~m}$ telescope at Las Campanas Observatory (Chile). PANIC uses a Hawaii $\mathrm{HgCdTe} 1024 \times 1024$ array with optics that give a scale of $0.125^{\prime \prime} /$ pix. We obtained 9 dithered frames in $J H K_{\mathrm{s}}$ separated $6^{\prime \prime}$, each with integration times 80, 60 and $30 \mathrm{~s}$, respectively, and with $120 \mathrm{~s}$ in the $\mathrm{H}_{2}$ filter. The total area covered by the combined frames was nearly 5.7 square arcminutes. The measured PSF ( FWHM) was about $0.8^{\prime \prime}$ in $J H K_{\mathrm{s}}$ and $0.7^{\prime \prime}$ in the $\mathrm{H}_{2}$ filter. The near-IR images were calibrated using the standard stars listed by Persson et al. (1998). Figure 1 shows a portion of the calibrated $J H K_{\mathrm{s}}$ and $\mathrm{H}_{2}$ images covering a $55^{\prime \prime} \times 55^{\prime \prime}$ field centered on [KG2001]102. We identified five optical stars from the DSS plates in the field. We used the USNO-B1.0 catalog positions to obtain astrometry on our images with an accuracy better than 0.5". [KG2001]102 appears in all bands as multiple, with three close but well separated stellar components. We named these A, B, and C, as labelled on the $J$ image presented in Fig. 1. A faint close fourth component (D) was also found after detailed analysis of the images.

Two direct CCD I-band images of the same region were obtained with IMACS (Inamori Magellan Areal Camera and

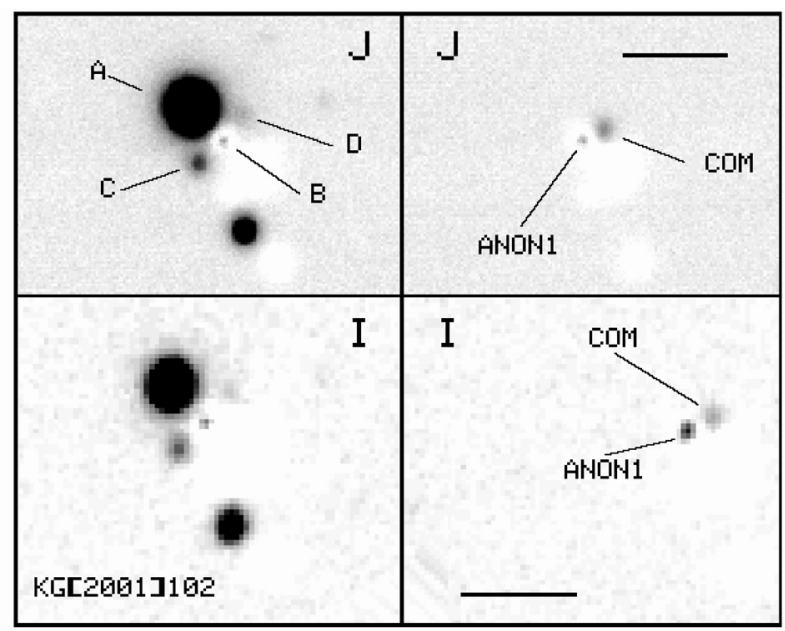

Fig. 2. Clay/PANIC J (top panels) and Baade/IMACS I (bottom panels) residual images of [KG2001]102 B and Anon1 that reveal the faint companions [KG2001]102 D and comAnon1. The horizontal bars are $5^{\prime \prime}$ long; the scale is the same for each filter. North is up and east is to the left. The measured separations and position angles are $2.23^{\prime \prime}$ and $225^{\circ}$ for $\mathrm{AB}, 1.64^{\prime \prime}$ and $130^{\circ}$ for $\mathrm{BC}, 1.42^{\prime \prime}$ and $321^{\circ}$ for $\mathrm{BD}$ and $1.10^{\prime \prime}$ and $295^{\circ}$ for Anon1 and its companion.

Spectrograph) in its direct imaging mode. Each was integrated under good seeing conditions for $60 \mathrm{~s}$. The scale was $0.222^{\prime \prime} /$ pixel. The measured stellar FWHM is $0.7^{\prime \prime}$. As no photometric standards were observed with this instrument, zeropoints were determined by averaging those values calculated using DENIS catalog entries for Anon $1(I=16.59)$ and of 


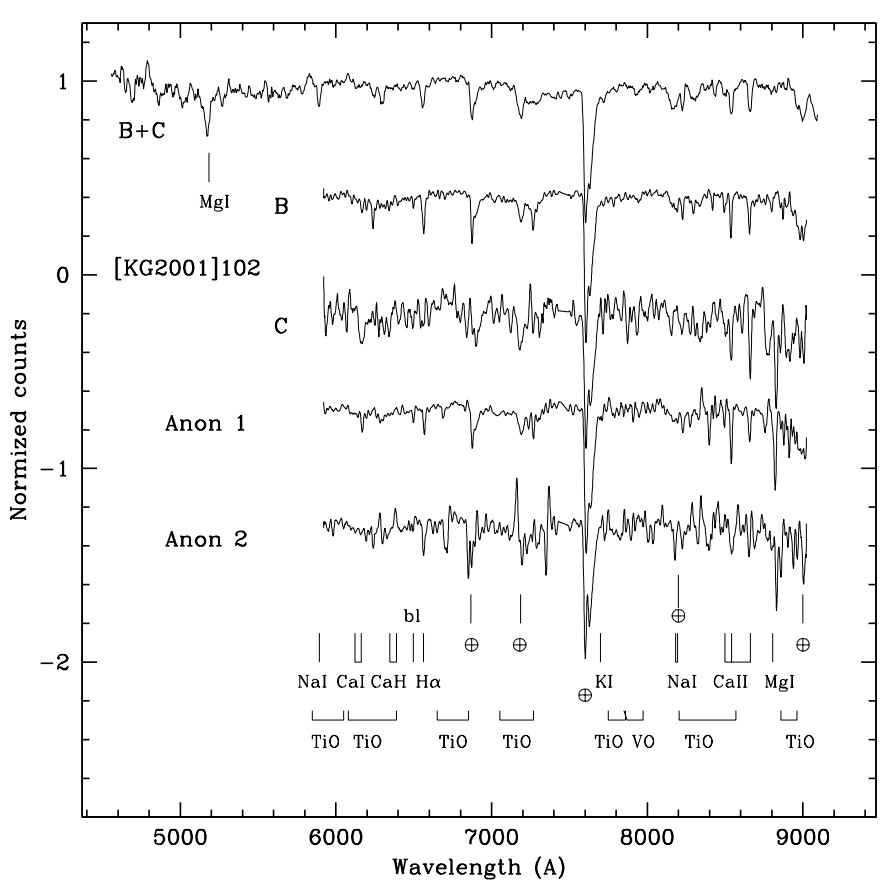

Fig. 3. Normalized spectra of [KG2001]102 B, [KG2001]102 C, Anon1, and Anon2. The combined spectrum labelled $\mathrm{B}+\mathrm{C}$ was taken with the duPont $2.5 \mathrm{~m}$ telescope while the individual spectra of $\mathrm{B}$, $\mathrm{C}$, Anon1 and Anon2 were taken with the Baade $6.5 \mathrm{~m}$ telescope. For clarity, these were shifted vertically. No flux calibration was performed.

[KG2001]102 $(I=15.64)$, the latter including the contribution from all four components.

A close examination of the $J, H$ and $K_{\mathrm{s}}$ (Fig. 1) and $I$ images reveals that the stellar profiles of two stars in the field, namely [KG2001]102 B and Anon1, appear elongated. In order to probe the meaning of this asymmetric profile, we subtracted the point-spread function (PSF) of a single star from the observed profile of the two elongated sources. We selected [KG2001]102 A as the best option for providing the "standard" PSF because it appears circular, is well separated from component B and is, at the same time, very close to it and not far from the star Anon1. It is also the brightest source in the field. Thus, a straight subtraction of a normalized and shifted image (to equate the fluxes of [KG2001]102 A to each of the target stars and to match their position) from the original one should result in a residual image that would expose any nearby extended emission or close companion. The left panels of Fig. 2 show the $J, I$ images for [KG2001]102 B while the right panels present the same for Anon1. In each of these two cases, a close faint companion is clearly seen to the NW, though the position angles differ for the two stars. In all $\mathrm{H}_{2}, J, H, K_{\mathrm{s}}$ frames and in the two $I$ independent frames, practically identical residuals were found. The positions of these companions, named [KG2001]102 D and comAnon1, were measured on all our subtracted frames and the mean values are listed in Table 1 together with their photometry also measured on the residual images. All four components lie within a radius of $\sim 2^{\prime \prime}$, while the separation of comAnon1 relative to Anon 1 is $1.10^{\prime \prime}$.
The same PSF-subtraction procedure was applied to the slightly fainter star located 13" south of [KG2001]102 B. Absolutely no trace of residual emission of a close companion is seen (only a slight mismatch of the PSFs at the center of the profiles, which appears in all cases) on any frame. Similar tests were performed on other faint stars in the field, confirming that no systematic instrumental effects are creating ghosts. It is important to note that, although the nearinfrared and the optical images were taken with different telescope/instruments combinations, the companions were present in all frames and that their measured positions coincided within $0.1^{\prime \prime}$ in all wavelengths.

Photometry of the sources detected in the field was performed using DAOPHOT (Stetson 1987) in IRAF $^{1}$ using the PSF fitting procedure. Table 1 gives the positions and $I J H K_{\mathrm{s}}$ photometry of the components of [KG2001]102 and other nearby sources labelled in Fig. 1. The accuracy of the $J H K_{\mathrm{s}}$ photometry is better than $0.03 \mathrm{mag}$, except for [KG2001]102 D and com-Anon 1 where the errors may be up to $10 \%$. For the isolated stars in our field that are also included in the $2 M A S S$ Point Source Catalogue, the JHK magnitudes agreed with our measurements within the quoted errors. The uncertainties in the $I$ photometry are estimated to be up to $10 \%$ because we used two very faint stars as local calibrators and one of them ([KG2001]102) contains a young and likely variable star (component A).

Comparing the $K_{\mathrm{s}}$ with the $\mathrm{H}_{2}$ images (see Fig. 1), we were unable to detect any $\mathrm{H}_{2}$ emission knots brighter than our sensitivity limit of $\sim 6 \times 10^{-32} \mathrm{~W} \mathrm{~m}^{-2} \mathrm{~Hz}^{-1} \operatorname{arcsec}^{-2}$.

\subsection{Optical spectroscopy}

In order to understand the nature of its components and to test whether [KG2001]102 is a physical system or caused by projection, two optical spectra of components $\mathrm{B}$ and $\mathrm{C}$ were obtained. The first, on the night of 29 November 2003 UT, using the WFCCD camera (http://www. lco.cl/lco/dupont/instruments/) on the duPont $2.5 \mathrm{~m}$ telescope at Las Campanas with the "blue" grism. This setting gives a nominal dispersion of $3 \AA /$ pix, with an approximate $7 \AA(F W H M)$ resolution and covering a useful wavelength range from $4800 \AA$ to $9200 \AA$. The $1.5^{\prime \prime}$ slit was oriented to include components $\mathrm{B}$ and $\mathrm{C}$ and so as to avoid the contribution of component A. Three separate spectra with a total integration time of 1700 seconds were gathered at an air mass of approximately 1.7 . The light of component B dominates the spectrum, as $\mathrm{C}$ is much fainter.

A second set of spectra was obtained on the night of 28 February 2004 UT, using the camera/spectrograph IMACS (http://www. lco.cl/lco/magellan/instruments/) mounted on the Magellan-Baade $6.5 \mathrm{~m}$ telescope. We used the "long camera" configuration with a $300 \mathrm{l} / \mathrm{mm}$ grating and a $1.2^{\prime \prime}$ slit width oriented along the line joining components $\mathrm{C}$

${ }^{1}$ IRAF is distributed by the National Optical Astronomy Observatory, which is operated by the Association of Universities for Research in Astronomy, Inc. under contract to the National Science Foundation. 
Table 1. Positions and near-IR photometry of the stellar sources.

\begin{tabular}{|c|c|c|c|c|c|c|}
\hline \multirow[t]{2}{*}{ Source } & $\overline{\alpha(J 2000)}$ & $\overline{\delta(J 2000)}$ & \multirow{2}{*}{$\begin{array}{c}I \\
\mathrm{mag}\end{array}$} & \multirow{2}{*}{$\begin{array}{c}J \\
\mathrm{mag}\end{array}$} & \multirow{2}{*}{$\begin{array}{c}H \\
\mathrm{mag}\end{array}$} & \multirow{2}{*}{$\begin{array}{c}K_{\mathrm{s}} \\
\mathrm{mag}\end{array}$} \\
\hline & $\mathrm{h} \mathrm{m} \quad \mathrm{s}$ & & & & & \\
\hline [KG2001]102 A & 110949.12 & -773120.5 & 16.14 & 13.09 & 12.46 & 11.86 \\
\hline [KG2001]102 B & 110948.63 & -773122.1 & 16.62 & 14.47 & 13.69 & 13.36 \\
\hline [KG2001]102 C & 110949.00 & -773123.1 & 19.82 & 17.60 & 16.67 & 16.29 \\
\hline$[\mathrm{KG} 2001] 102 \mathrm{D}$ & 110948.36 & -773121.0 & 21.4 & 18.8 & 17.9 & 17.4: \\
\hline Anon1 & 110944.9 & -773107 & 16.74 & 14.66 & 13.89 & 13.40 \\
\hline com-Anon1 & 110944.6 & -773106 & 20.7 & 18.3 & 17.5 & 17.0 \\
\hline Anon2 & 110953.3 & -773140 & 18.59 & 16.34 & 15.53 & 15.37 \\
\hline
\end{tabular}

Table 2. Line equivalent width measurements for the component B and the star Anon1.

\begin{tabular}{llll}
\hline \hline Element & $\begin{array}{l}\text { Lambda } \\
(\AA)\end{array}$ & $\begin{array}{l}E W(\mathrm{~B}) \\
(\AA)\end{array}$ & $\begin{array}{l}E W(\text { Anon1 }) \\
(\AA)\end{array}$ \\
\hline $\mathrm{Mg} \mathrm{I}$ & 5173 & 6.7 & \\
$\mathrm{Na} \mathrm{I}$ & 5893 & 2.3 & \\
$\mathrm{Ca}$ I & 6162 & 1.31 & \\
$\mathrm{CaH}$ & $6346 / 6389$ & $0.32 / 0.22$ & 1.62 \\
$\mathrm{H} \alpha$ & 6563 & 3.58 & \\
$\mathrm{Li} \mathrm{I}$ & 6708 & $0.10 ?$ & \\
$\mathrm{Na} \mathrm{I}$ & $8183 / 8195$ & $1.01 / 0.81$ & \\
$\mathrm{Ca}$ II & $8498 / 8542 / 8662$ & $1.37 / 3.29 / 3.16$ & $1.39 / 4.23 / 2.86$ \\
$\mathrm{Mg} \mathrm{I}$ & 8807 & 0.83 & \\
\hline
\end{tabular}

and B (SE - NW). Serendipitously, two other stars in the field (marked Anon1 and Anon2 in Fig. 1) lay on the slit. The nominal dispersion is $0.74 \AA$ A pixel and the measured resolution was $4 \AA$ (FWHM). The wavelength coverage was $5950 \AA$ to $9020 \AA$ with a $60 \AA$ gap at $7470 \AA$. Thanks to good seeing, the spectra of components $\mathrm{B}$ and $\mathrm{C}$ were clearly separated and could be extracted independently though the latter, being three magnitudes fainter $(I=19.9)$, had a poor signal to noise.

Wavelength calibrations were performed with a $\mathrm{He}-\mathrm{Ne}$ lamp obtained between two stellar spectra. The data were reduced in the usual way, including sky subtraction but no flux calibration was attempted. The residual sky-lines were removed by interpolating the signal at neighboring points.

Figure 3 shows the normalized duPont spectrum of component $\mathrm{B}$ and $\mathrm{C}$ as well as the Baade individual spectra of each star. In addition, the spectra corresponding to Anon1 and Anon 2 are also shown. Table 2 reports the measured equivalent widths for several lines in the spectrum of component B. For Anon 1 the equivalent widths of $\mathrm{H} \alpha$ and the calcium triplet are also listed.

\section{Discussion}

\subsection{Spectral classification}

Of the two independent spectra of [KG2001]102, the one taken with WFCCD/duPont with lower spatial and spectral resolution and with higher wavelength coverage includes the added light of components $\mathrm{B}, \mathrm{C}$ and $\mathrm{D}$ but by far the brightest $\left(\Delta I_{\mathrm{BC}}>3\right)$ of these (B) dominates. In the IMACS/Baade observations, it was possible to separate the spectrum of component $\mathrm{C}$ from that of component B (see Fig. 3). The main absorption lines of $\mathrm{H} \alpha$, Ca I, Ca II, Na I and Mg I are clearly seen in both spectra of [KG2001]102 B. By comparing these spectra with the grids by Allen \& Strom (1995), Kirkpatrick et al. (1991) and Torres-Dodgen \& Weaver (1993), we were able to assign a spectral type K7V to [KG2001]102 B with an estimated uncertainty of up to three subtypes. This large uncertainty is due to the fact that neither spectrum was flux-calibrated or corrected for telluric absorption and taken with relatively high sky emission. The spectrum of [KG2001]102 C shows the Ca II triplet in absorption and no $\mathrm{H} \alpha$ line but, due to its low signal to noise ratio, we were unable to unambiguously assign a spectral type, although a late M-type is discarded as no deep TiO bands are seen.

Finally, the spectrum of Anon1 looks very similar to that of [KG2001]102 B, while the spectrum of the fainter star Anon2 is too noisy to attempt a spectral type determination.

\subsection{Magnitudes and colors}

Near-infrared color-color and magnitude-color diagrams are useful tools to understand the nature of the sources detected in photometric surveys. Figure 4 a shows the $J-H$ vs. $H-K_{\text {s }}$ and Fig. $4 \mathrm{~b}$ the $K$ vs. $H-K$ diagrams of the seventeen sources that we detected in an area of $55^{\prime \prime} \times 55^{\prime \prime}$ around [KG2001]102. The filled circles represent the four stellar components of [KG2001]102, all listed in Table 1. Star [KG2001]102 A shows significant near-IR excess emission, probably originating in a circumstellar dust envelope or disk. The stars with $J-H$ colors larger than unity, implying $E(J-H)>0.38$ or $A_{V}>3.7$, are most probably background stars.

In the case of component $\mathrm{C}$, if it were an MOV star, the latest possible given that only very weak $\mathrm{TiO}$ bands can be traced in its noisy spectrum (see Fig. 3), its distance and extinction would be between 1.6 and $2.3 \mathrm{kpc}$ and $A_{V}=2.5$; other possible solutions include, eg., a G8V with $A_{V}=5$ and $d=2.3 \mathrm{kpc}$. No realistic solution exists for an evolved star (luminosity class III, II or I) for this or any of the other stars reported here as, in all cases, their distances from the Sun would be well in excess of $20 \mathrm{kpc}$, outside the Milky Way.

A group of stars with $J-H$ around 0.75 , which includes [KG2001]102 B and Anon1 appear to form a set of main sequence M 2-M 5 stars reddened by the same $E(J-H) \simeq 0.17$, corresponding to $A_{V}=1.5$, as shown in Fig. 4a. However, in Sect. 3.1 we estimated a K7V spectral type for [KG2001]102 B and Anon1. Assuming for this spectral type an intrinsic color 

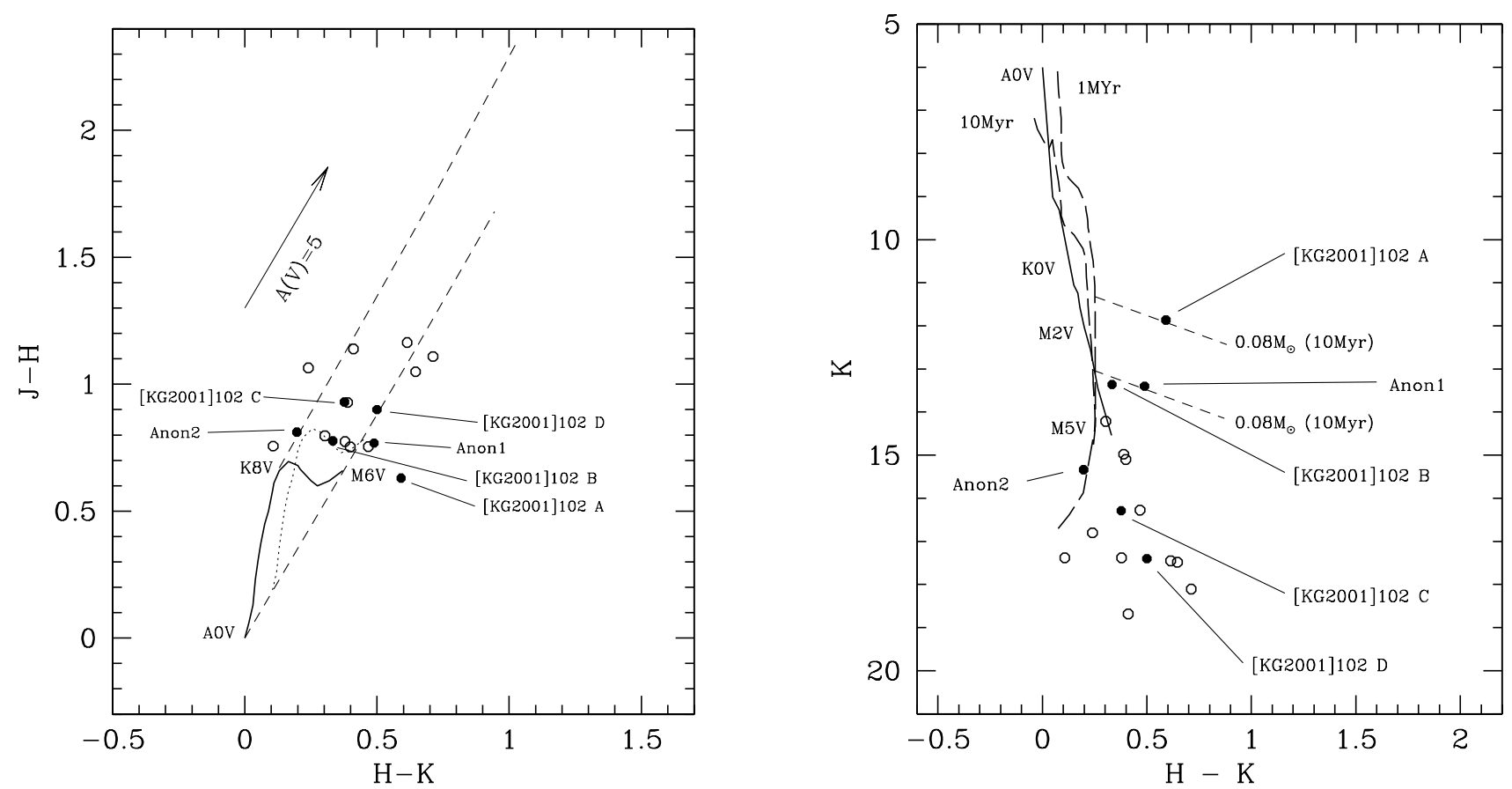

Fig. 4. $a$. Left panel: $J-H$ vs. $H-K_{\mathrm{s}}$ diagram of the sources detected. The solid line marks the locus of the main sequence (Bessel \& Brett 1988), while the dashed lines define the standard reddening vector (Rieke \& Lebofsky 1985). The length of the arrow represents $A_{V}=5$. The dotted line represent the main sequence reddened by $E(J-K)=0.23$. b. Right panel: $K_{\mathrm{s}}$ vs. $H-K_{\mathrm{s}}$ diagram. The solid line represents the ZAMS and the long-dashed lines indicate the $1 \mathrm{Myr}$ and $10 \mathrm{Myr}$ isochrones for low mass stars and brown dwarfs from the models by D'Antona $\&$ Mazzitelli (1997). Reddening lines for $A_{V}=10$ (short-dashed lines) are drawn for a $0.08 M_{\odot}$ objects of 1 and 10 Myr. Filled circles represent the sources listed in Table 1.

index $(J-H)_{\circ}=0.66$ and absolute magnitudes $M_{J}=5.7$, $M_{K}=4.9$ (Bessell \& Brett 1988; Schmidt-Kaler 1983), their distances from the Sun would be 550-700 pc, which would place them behind the Cha I cloud, compatible with the absence of signposts of chromospheric activity in its spectrum. Their color excess would be $E(J-H) \simeq 0.13$, which corresponds to $A_{V} \simeq 1.2$. This is surprising because background stars are expected to be more highly reddened than any star member of the cloud. According to the extinction map of the Cha I cloud by Cambrèsy et al. (1997), the mean total extinction caused by the cloud at the position of our field is in the range $3.0<A_{V}<3.5$. As both, our analysis and Cambrèsy et al.'s study, are based on $J$ magnitudes, the argument is independent of the reddening law assumed. We tested the possibility of a local dust column density inhomogeneity in the cloud at the position of [KG2001]102, that is, the presence of a "hole" of diameter of a less than one thousand AU (a few arcseconds) that would permit the detection of a number of background stars with extinctions lower that the local average. We performed star-count analyses on $J, H$ and $K$ 2MASS images centered on [KG2001]102 and found no evidence of the existence of such local lower-extinction region.

Probably the answer to this dilemma lies in the incorrect assumption that the intrinsic $J H K$ colors of these stars correspond to those indicated by their spectral type. Indeed, the location of these stars on the $J-H$ vs. $H-K$ diagram is incompatible with their spectra unless they have strong $K$-band excess emission, a possibility that contradicts the fact that these stars display $\mathrm{H} \alpha$ line in absorption, not in emission. We also compared the location of our program sources on several twocolor diagrams with that of stars and brown dwarfs classified as members and non-members of Cha I in a large-scale survey. In Fig. 5 we present the $I-J$ vs. $J-H$ diagram of the sources studied by Luhman (2004) in the direction of Cha I with $J>12$ (we omit the brightest stars to avoid overcrowding, though the trends are the same when all stars are included). A segregation between members and non members of the cloud is evident. Luhman (2004) used 2MASS and DENIS photometric values for his study, thus allowing direct comparisons with our photometry. He also applied several criteria (mostly spectroscopic) to differentiate between members from non-members. It is intriguing that our program stars in Table 1 occupy an area defined by the young cloud members and seem to lie away from those areas occupied by background and foreground stars on this diagram. This characteristic is also evident in the $J-H$ vs. $H-K$ and other two-color plots. Clearly, [KG2001]102 A and $\mathrm{D}$ share the characteristics of young members of the Cha I cloud while less strict conclusions can be derived from the location of components $\mathrm{B}$ and $\mathrm{C}$ and Anon1 in these plots. The explanation for these effects $-I J H K$ photometry in disagreement with a normal photosphere of a given spectral type plus interstellar reddening- is unclear. A few other sources originally proposed to be substellar objects in young regions based on photometry turned out to have spectra of earlier-type stars (see e.g., Martin 2000).

It is important to notice that our $J H K$ magnitudes for isolated sources in the field compare well with $2 M A S S$ and DENIS data (with differences $<0.03$ mag. in all bands) 


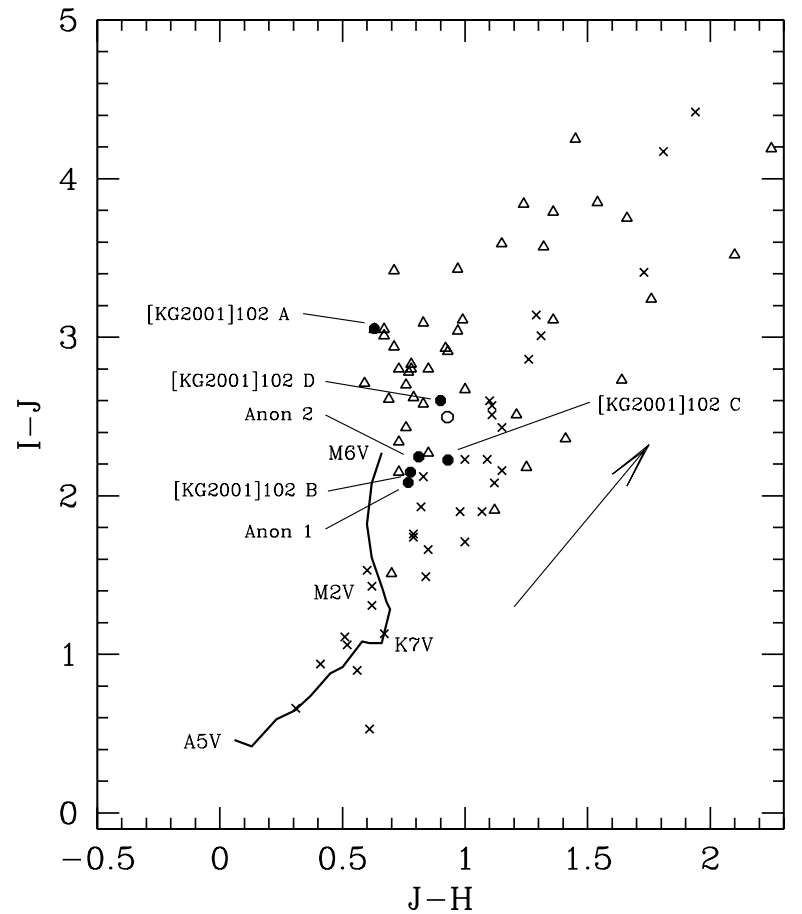

Fig. 5. $I-J$ vs. $J-H$ diagram of sources in the direction of Cha I. The solid line marks the locus of the main sequence (Bessel \& Brett 1988). The arrow represents the standard reddening vector (Rieke $\&$ Lebofsky 1985) with length $A_{V}=5$. Filled circles represent the sources listed in Table 1, open triangles and crosses are objects with $J>12$ classified as members and non-members of the cloud, respectively, by Luhman (2004) with $J H K_{\mathrm{s}}$ from $2 M A S S$ and $I$ from DENIS.

and thus the discrepancies between the derived spectral types and the $J-H$ and $H-K$ colors cannot be ascribed to observational errors.

One basic question regarding the enigma poised by [KG2001]102 concerns the probability of this being a physical system or rather an illusion created by a chance projection effect. First we measured the projected density $N(J)$ of stars brighter than a certain magnitude $J$ in this direction over an area centered on [KG2001]102 which is large enough to yield statistically valid results and small enough to safely assume that the $A_{V}$ is constant. For this, we obtained $J$ photometry for all sources in the total area surveyed $\left(2 \times 10^{4}\right.$ square arcseconds) and measured the cumulative number density distribution, $N(J)$, as a function of $J$ magnitude. The probability of finding a chance companion of magnitude $J$ at a distance $r$ from any star, eg. [KG2001]102 A, would be $\pi r^{2} N(J)$. The computed probability of randomly finding a companion like component $\mathrm{B}$ is $1.5 \times 10^{-3}$ while for a triple optical like $\mathrm{ABC}$, it is $3.8 \times 10^{-5}$ and an order of magnitude lower for ABCD. There is, therefore, a very high probability that at least three of these stars form a physical multiple system or, in different words, it is highly improbable that components $\mathrm{B}$ and $\mathrm{C}$ and $\mathrm{D}$ are all background stars.

Figure $4 \mathrm{~b}$ shows the $K_{\mathrm{s}}$ vs. $H-K_{\mathrm{s}}$ diagram of the observed sources together with the evolutionary tracks of D'Antona \& Mazzatelli (1997) computed for the distance of Chamaeleon. [KG2001]102 A lies very close to the limits of a sub-stellar object of mass $0.08 M_{\odot}$ (dashed lines in Fig. 4b) for ages $<10^{7}$ years. The location of [KG2001]102 $\mathrm{C}$ and [KG2001]102 D in this diagram may also be consistent with the possibility that these are young brown dwarfs.

\subsection{Luminosities, masses and ages}

A new determination of bolometric luminosity, mass and age is obtained for the young member of Cha I, [KG2001]102 A, using the near-IR photometry of Table 1, and the classical relationships adopted by Saffe et al. (2003):

$\log L_{\mathrm{bol}} / L_{\odot}=1.89-0.4 M_{\mathrm{bol}}$,

$M_{\mathrm{bol}}=J-A_{J}-\mathrm{DM}+\mathrm{BC}_{J}$,

$A_{J}=2.63 E(J-H)$,

where $\mathrm{DM}=6$ is the distance modulus for the Cha I dark cloud, and $\mathrm{BC}_{J}$ is the $J$-band bolometric correction, which we took from Wilking et al. (1999) together with the $T_{\text {eff }}$ corresponding to the spectral type and the corresponding intrinsic colors. The position of the source on the $L_{\text {bol }} / L_{\odot}$ vs. $T_{\text {eff }}$ was then compared with the evolutionary models of D'Antona \& Mazziteli (1997), Baraffe et al. (1998) and Burrows et al. (1997) to obtain its mass and age. These values are shown in Table 3. Although the implied mass varies somewhat with the model assumed, the sub stellar nature of [KG2001]102 A is confirmed.

\subsection{Spectral energy distribution}

We obtained the spectral energy distribution (SED) of the YBD [KG2001]102 A by combining the optical photometry taken from the USNO-B1 catalog, and our near infrared magnitudes. This source was not detected by ISOCAM at the quoted sensitivity for this region (see Persi et al. 2000). Figure 6 shows the SED. The arrows in Fig. 6 indicate the ISOCAM upper limits at 6.7 and $14.3 \mu \mathrm{m}$. We compared the spectral points with a de-reddened black body computed assuming the effective temperatures, $T_{\text {eff }}$, of this object as derived by Saffe et al. (2003) (solid lines in Fig. 6). The agreement is good, except for the spectral point at $2.2 \mu \mathrm{m}$ that indicates a slight IR excess, as previously mentioned in Sect. 3.2. Observations at higher sensitivity with the Spitzer Telescope should tell us whether this young sub-stellar object has mid-IR excesses as observed in a few other YBDs and ascribed to the presence of a circumstellar dust disk (Natta \& Testi 2001).

\section{Conclusions}

We presented sub-arcsec resolution and high sensitivity images taken with $I J H K_{\mathrm{S}}$ and $\mathrm{H}_{2}$ filters of a region of the Chamaeleon I dark cloud that contains the candidate sub-stellar object [KG2001]102 complemented with red spectra of four sources in the close vicinity of [KG2001]102. From the analysis of the images and spectra, the following conclusions can be derived:

1) We found that [KG2001]102 is composed of four sources, all within a radius of $2^{\prime \prime}$. The brightest component $\mathrm{A}$ 
Table 3. Derived parameters at $d=160$ pc.

\begin{tabular}{lcccccccc}
\hline \hline Object & $\log T_{\text {eff }}$ & $\log L / L_{\odot}$ & $M / M_{\odot}^{1}$ & $\operatorname{age}^{1}(\mathrm{Myr})$ & $M / M_{\odot}^{2}$ & age $^{2}(\mathrm{Myr})$ & $M / M_{\odot}^{3}$ & age $^{3}(\mathrm{Myr})$ \\
\hline$[$ KG2001]102 A & 3.44 & -1.71 & 0.054 & 3 & 0.040 & 0.1 & 0.033 & 1 \\
\hline
\end{tabular}

${ }^{1}$ Models by D'Antona \& Mazziteli (1997). ${ }^{2}$ Models by Baraffe et al. (1998). ${ }^{3}$ Models by Burrows et al. (1997).

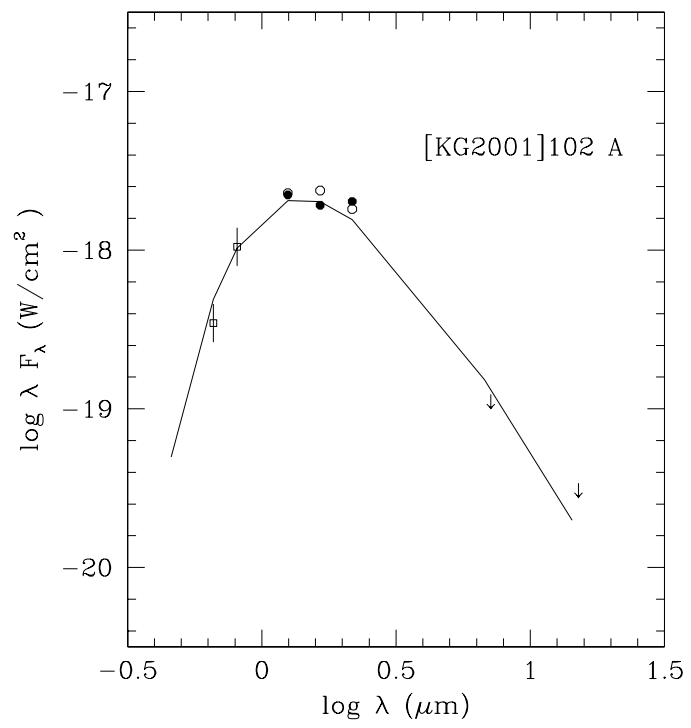

Fig. 6. Spectral energy distribution of the source [KG2001]102 A in Cha I. Filled circles are our NIR observations, open circles are the 2MASS flux densities. Open triangles are the photometric points of Kenyon \& Gomez (2001). The arrows indicate upper limits of the ISOCAM observations at 6.7 and $14.3 \mu \mathrm{m}$. Open squares are from the USNO-B1 catalog. The solid line represents a black body at the effective temperature of [KG2001]102 A reddened by $A_{V}=1.0$.

is a young sub-stellar object with a mass in the range 33-55 $M_{\text {Jup }}$ and showing a significant infrared excess at $2.2 \mu \mathrm{m}$.

We classified the spectrum of component B as K7V. This spectral type, combined with the observed $J$ and $J-H$, suggest that this object is a background star located at a distance of $\sim 550 \mathrm{pc}$ with $A_{V} \sim 1.2$, a much lower extinction than that caused by the cloud in this location $\left(A_{V}>3\right)$. Furthermore, a K7V spectral type seems difficult to reconcile with the measured $I J H K$ colors of this star, which resemble those of reddened $\left(A_{V} \sim 1\right)$ late-M members of the Cha I. Near-IR colors of component $C$ and D are also compatible with those of young members of Cha I, although our noisy spectrum of component $\mathrm{C}$ seems to exclude this possibility. However the poor signal to noise spectrum available for the component $C$ and the lack of spectroscopic information on component $\mathrm{D}$ preclude us from knowing the true nature of these objects.

Using star counts in the $J$ band in our field, we calculated the probability of finding by chance projection a double star like A and B. It was found to be $1.5 \times 10^{-3}$ and $3.8 \times 10^{-5}$ for a triple optical system like $\mathrm{ABC}$ and an order of magnitude lower for a projected quadruple which includes D. Therefore, it seems highly improbable that the multiple appearance of this object is caused by projection.
The explanations for these apparent contradictions in the observational evidence are not understood and also explain why other candidate YBDs that have been proposed based on photometry alone turn out to show spectra of earlier-type atmospheres.

2) We also report the detection of a faint close companion (separation 1.1" and $\Delta J=3.7$ ) to the star Anon 1. This last object has similar spectral characteristics as component B.

3) Our $\mathrm{H}_{2}$ image does not show the presence of any shocked gas structure in the vicinity of any star in the area surveyed.

Acknowledgements. We are grateful to Las Campanas staff for efficient assistance during the observing runs. M.G. and P.P. also acknowledge financial support form the bilateral project SECyT(Argentina)-MAE(Italy) $21 \mathrm{~F}$ and MT acknowledges UNAMDGAPA grant IN-102403.

\section{References}

Allen, L. E., \& Strom, K. M. 1995, AJ, 109, 1379

Baraffe, I., Chabrier, G., Allard, F., \& Hauschildt, P. H. 1998, A\&A, 337,403

Bate, M. R., Bonnell, I. A., \& Bromm, V. 2002, MNRAS, 332, L65

Bessel, M. S., \& Brett, J. M. 1988, PASP, 100, 1134

Burrows, A., Marley, M., Hubbard, W. B., et al. 1997, ApJ, 491, 856

Cambrèsy, L., Epchtein, N., Copet, E., et al. 1997, A\&A, 324, L5

Comerón, F., Neuhäuser, R., \& Kaas, A. A. 2000, A\&A, 359, 269

D’Antona, F., \& Mazzitelli, I. 1997, MSocAItal, 68, 807

Kenyon, S. J., \& Gómez, M. 2001, AJ, 121, 2673

Kirkpatrick, J. D., Henry, T. J., \& McCarthy, D. W. Jr. 1991, ApJS, 77, 417

Klein, R., Apai, D., Pascucci, I., Henning, Th., \& Waters, L. B. F. M. 2003, ApJ, 593, 57

Luhman, K. 2004, ApJ, 602, 816

Martin, E. L. 2000, AJ, 120, 2114

Natta, A., \& Testi, L. 2001, A\&A, 376, L22

Neuhäuser, R., Brandner, W., Alves, J., Joergens, V., \& Comerón, F. 2002, A\&A, 384, 999

Pascucci, I., Apai, D., Henning, Th., \& Dullemond, C. B. P. 2003, ApJ, 590, L111

Persi, P., Marenzi, A. R., Olofsson, G., et al. 2000, A\&A, 357, 219

Persson, S. E., Murphy, D. C., Krzeminski, W., et al. 1998, AJ, 116, 2475

Reipurth, B., \& Clarke, C. 2001, AJ, 122, 439

Rieke, G. K., \& Lebofsky, M. J. 1985, ApJ, 288, 618

Saffe, C., Gómez, M., Randich, S., et al. 2003, A\&A, 409, 993

Schmidt-Kaler Th. 1983, In Landolt-Börnstein, Neue Ser., p. 1, Gr VI, Vol. 2b, Stars and Star Clusters, ed. K. Schaifers \& H.H. Voight (Berlin: Springer), 10

Stetson, P. B. 1987, PASP, 99, 191

Torres-Dodgen, A. V., \& Weaver, W. B. 1993, PASP, 105, 693

Wilking, B. A., Greene, T. P., \& Meyer, M. R. 1999, AJ, 117, 469 